Genésio Vicentin 1

Augusto Hasiak Santo 2

M arília Sá Carvalho 3

\section{Mortalidade por tuberculose e indicadores sociais no município do Rio de Janeiro}

\author{
Tuberculosis mortality and social indicatory \\ in Rio de Janeiro city
}

1 Centro de Estudos da Saúde do Trabalhador e Ecologia Humana/Cesth, Escola Nacional de Saúde Pública, Fundação Oswaldo Cruz. Rua Leopoldo Bulhões 1480, Manguinhos, 21041-210,

Rio de Janeiro RJ. vicentin@ensp.fiocruz.br 2 Departamento de Epidemiologia da Escola de Saúde Pública da Universidade de São Paulo. 3 Departamento de Epidemiologia da Escola Nacional de Saúde Pública. Fundação Oswaldo Cruz.

\begin{abstract}
Regional tuberculosis mortality levels and their Rio de Janeiro City principals social indicatories at year 1991 have been compared in this study. Software SPSS 8.0 - 1997 was applied on making statistical data analysis, and have disclosed a present correlation among most indicative socioeconomics trends and the annual tuberculosis mortality coeficient/100 thousand inhabitants. Others social indicatives than Robin $\mathrm{H}$ ood index, $10 \%$ more rich to $40 \%$ more poor reason, head of family proportion wich 1-2 minimum wage monthly gain, disclosed imediate and significative statistical assurance level. In reverse and significative statistical assurance level, resident proportion with more 10 years old that have superior course, house área average, room average from house, minimum wage average from head family and head family proportion with have 10-15, 16-20 and 20 or more minimum wage gain. The class distribution of this indicative and its presentation in a M unicipal maps, for Administratives Regions, disclose multiple Rio de Janeiro existense when we looks them by a social perspective: of the disease focus or others indicative. Key words M ortality, Tuberculosis, Social indicatory
\end{abstract}

Resumo Este artigo estuda a mortalidade por tuberculose no município do Rio de Janeiro ocorrida no ano de 1991 em comparação com indicadores sociais. U tilizou-se o software SPSS 8.0 - 1997 na análise estatística dos dados, que mostrou a existência de correlação entrea maioria dos indicadores socioeconômicos estudados e o coeficiente de mortalidade por tuberculose por 100 mil habitantes/ano. Apresentaram correlação significativa e direta os indicadores: índice de Robin Hood, razão de renda entre os 10\% mais ricos e os $40 \%$ mais pobres e proporção de chefes de família com renda média entre um e dois salários mínimos. A correlação foi inversa e significativa com os indicadores: proporção de residentes com mais de 10 anos com curso superior, área média por domicílio, número de cômodos por domićlio, renda média em salários mínimos, e proporção de chefes de família com rendas entre 10-15, 16-20 e acima de 20 salários mínimos. Para a apresentação destes indicadores usou-se mapas do município, categorizados e distribuídos por Regiões Administrativas, revelando distintos Rios de Janeiro, quando esteévisto sob o ângulo social: da doença, ou de vários outros indicadores.

Palavras-chave M ortalidade, Tuberculose, Indicadores sociais 


\section{Introdução}

A busca da relação entre componentes da vida social ea morbi-mortalidade é um caminho freqüentemente trilhado pelos estudos epidemiológicos, interessando principalmente aqueles referentes às condições materiais de vida. Alguns desses estudos são bastante conhecidos, podendo ser incluídos na própria história da epidemiologia. Um exemplo clássico foi o estudo de Snow (1967) sobre a epidemia de cólera em Londres, ocorrida em meados do século 19. Neste estudo, o autor estabeleceu a pertinência do contexto epidemiológico ao processo histórico-social, ao relacionar o encadeamento deste ao surgimento das epidemias de cólera nas colônias do Império Britânico e em Londres.

Assim como a cólera, a tuberculose é outra doença transmissível cujo processo saúde-doença está em estreita rel ação de determinação com o desenvolvimento histórico social. Estabelece-se uma relação à qual o processo particular da tuberculose pertence e tem como principal determinante as condições sociais de vida. Estudos epidemiológicos, realizados em países europeus e nos Estados Unidos, demonstraram a vinculação entre o processo histórico da sociedade e o particular dessa doença, o que lhe confere também caráter histórico (Dubos \& Dubos, 1952; M ckeown \& Lowe, 1981; Black et al., 1982; Engels, 1985; Stead et al., 1990; Bates, 1994).

No Brasil, aquela relação foi demonstrada por estudos como os de Albuquerque \& Rodrigues (1952), Ribeiro (1956), Ruffino Netto (1975), Ruffino Netto e Pereira (1981), Pereira e Ruffino N etto (1985), Guimarães (1985), Penna (1988a, 1988b, 1988c), Almeida (1990), Lima (1996), Bertolli Filho (1997); eAntunes e Waldman (1999). M ais recentemente, N ascimento (1991) eVicentin (2000) analisaram a participação institucional no combateà doença e mostraram que as diferentes orientações da estratégia política e econômica, em períodos historicamente distintos, condicionaram o papel do Estado frente a al guns dos mais relevantes problemas sanitários do país, sen do o principal determinante no processo de controle da tuberculose.

No terreno próprio do processo saúde-doença, hoje, há consenso sobre a relação entre as condições sociais de vida e o desenvolvimento de doenças. Embora ainda haja controvérsia sobre o modo como se dá o enlace entre o componente social da vida e o substrato biológico, cresce a necessidade de identificar indicadores específicos de condições de vida, que se asso- ciem ao desenvolvimento de determinadas doenças ou padrões de morbidade e mortalidade. Assim, é bastante apropriado o estudo da associação entre indicadores pertencentes às esferas biológica e social no desenvolvimento de processos particulares de doenças determinadas, como a tuberculose. Da mesma forma, é relevante a quantificação da força dessas associações mediante procedimentos estatísticos.

Além disso, alguns indicadores sociais têm para a saúde pública uma dupla performance. Uma delas, de forma indireta, indicando a própria condição de vida da população em pauta, constitui os determinantes concretos de sua morbi-mortalidade e permite seu agrupamento em categorias mais ou menos homogêneas, diferenciadas socialmente. Como a condição social é determinante do padrão epidemiológico (Black et al., 1982; César, 1990; Vicentin, 1991) surge, em segundo lugar, a possibilidade de se identificar associações desses indicadores sociais com processos particulares de saúde-doença, representados por sua morbi-mortalidade (os indicadores do substrato biológico). Esteúltimo tipo de associação, que se dá no nível mais imediato das relações, é o próprio processo concreto de saúde-doença, representado pelos processos particulares como a tuberculose.

Assim, foi possível tomar como estratégia de aproximação o uso de procedimentos estatísticos que transformam as associações supostas em produtos mensuráveis: como uma freqüência determinada ou uma probabilidade de vínculo ou enlace entre os componentes relacionados. Como objetivo imediato da aplicação da metodologia no caso da tuberculose, que compõe um universo bem conhecido e com indicadores definidos para tais abordagens cognitivas, quantificou-se e analisou-se o comportamento de tais indicadores e sua relação com a mortalidade por tuberculose. Discutiu-se também a propriedade e a adequação de tais indicadores sociais para o estudo do desenvolvimento da tuberculose e a condição de um indicador formado por um componente biológico, o "coeficiente de mortalidade por tuberculose", desempenhar um papel de indicador social. No processo particular de saúde-doença da tuberculose, a mortalidade constitui um dos indicadores de saúde que mais traduz o enlace do social com o biológico. Por ser esta uma doença crônica com ampla possibilidade de cura, permite ir um pouco mais além nessa vertente de exploração. Traduz principal mente os efeitos da ação do componente institucional, sendo um bom indicador da 
eficiência do aparato destinado ao trabalho de controle da doença e da operação do mesmo (Albuquerque \& Rodrigues, 1952; Almeida, 1990; Bates, 1994; Antunes \& Waldman, 1999).

\section{Metodologia}

Desenho do estudo - Foi feito um estudo ecológico estimando a correlação entre pares de indicadores (Vieira, 1985; Spiegel, 1985). As variáveis socioeconômicas testadas foram selecionadas em função de seu potencial explicativo sobre a mortalidade e a prevalência da tuberculose, seja em sentido direto ou inverso.

A unidade territorial de análise, definida por motivos de disponibilidade de dados e continuidade histórica, foi a Região Administrativa do Município do Rio de Janeiro.

O programa TabW in foi utilizado para a elaboração dos mapas, onde os indicadores foram divididos em cinco classes iguais. $\mathrm{Na}$ estimativa da correlação foi utilizado o SPSS 8.0 TM .

Ano de estudo e fonte dos dados - 0 ano estudado foi o de 1991, por ser ano censitário, utilizando a divisão territorial, vigente desde 1979, e para a qual se dispunha dos dados dos óbitos, embora a estrutura administrativa estivesse sendo alterada.

Os óbitos, codificados segundo as regras da Nona Revisão da Classificação Internacional de D oenças pel os códigos 010 a 018, foram obtidos dos dados produzidos pelo Subsistema de Informação de M ortalidade (SIM).

Utilizaram-se indicadores socioeconômicos do Instituto de Planejamento Municipal (IPLAN RIO, 1991, 1992, 1993/1994 e 1995) e do estudo de Szwarcwald et al. (1999).

Área de estudo - 0 município do Rio deJaneiro dividido em vinte e quatro Regiões Administrativas (RAs) e seis Áreas de Planejamento (APS) (Quadro 1) (IPLAN RIO, 1979; 1980/ 1981; 1980/1984; 1991; 1993 e 1995; Fundação IBGE, 1980 e 1991).

Indicadores - Foi utilizado o coeficiente de mortalidade por tuberculose, por 100 mil habitantes/ano (CM TB) calculado para cada uma das RAs e as seis APs. Os demais indicadores utilizados são especificamente sociais ou econômicos e foram agrupados segundo estas características. Mas, mesmo os econômicos embutem forte conotação social, como determinantes do processo saúde-doença estudado.

Indicadores sociais: a) taxa de analfabetos (TA) - proporção de chefes de domicílio sem instrução (Szwarcwald et al., 1999); b) índice de pobreza (IP) - proporção de chefes de domicílio com rendimento mensal menor do que um salário mínimo (Szwarcwald et al., 1999); c) densidade demográfica (DD) - transformação logarítmica do número de habitantes/hectare (Szwarcwald et al., 1999); d) densidade de população favelada (DPF) - transformação logarítmica do número de habitantes residentes em setores censitários classificados como "aglomerados subnormais" por hectare (Szwarcwald et al., 1999); e) taxa de escolaridade de nível superior (TES) - proporção da população com mais de dez anos com curso superior completo (IPLANRIO, 1991); f) área média por domicílio (AMD) - área média construída por domicílio, em metro quadrado (IPLANRIO, 1992); g) número médio de cômodos (NMC) - média de cômodos por domicílio (IPLANRIO, 1995).

Indicadores de renda: $h$ ) índice de Robin Hood (IRH) - indica a proporção de renda que deveria ser retirada dos ricos e transferida para os pobres para que se obtivesse distribuição eqüitativa da renda. Para seu cál culo, deve-se obter a renda percentual de cada decil em relação à renda total. Subentendendo-se a renda de $10 \%$ para cada decil, caso a distribuição fosse eqüitativa, sempre que ultrapassar corresponderá a um excesso a ser redistribuído. A soma desses excessos corresponde a um percentual que é o IRH (Szwarcwald et al., 1999); i) índice de Gini (IG) - uma medida da concentração de renda, que varia de zero a um, e este último valor corresponde à desigual dade máxima. "É derivado por meio da curva de Lorenz, gráfico que representa os percentuais de renda acumulada por decil da população". A fórmula para seu cálculo pode ser obtida em Hammond e M ccullagh (1978), citados por Szwarcwald et al., (1999); j) razão da renda média entre os 10\% mais ricos e os $40 \%$ mais pobres $(r 10 \%+)$. É calculado dividindo-se a renda total do último decil pela dos 40\% mais pobres (Szwarcwald et al., 1999); k) renda média (RM ) - renda média dos chefes de domicílio em salários mínimos (IPLAN RIO, 1995); I) renda média até meio salário mínimo (sm) (r1/2), renda média entre meio e um sm (r1/2-1), renda média entre um e dois sm ( $r 1-2)$, renda média entre dez e quinze sm (r10-15), renda média entre quinze e vinte sm (r15-20) e renda média acima de vinte sm ( $r 20 \mathrm{e}+)$ - rendimento médio dos chefes de família situado nas faixas de sm acima (IPLAN RIO, 1995). 
Quadro 1

Áreas de Planejamento e Regiões Administrativas do município do Rio de Janeiro (1979-1990).

\begin{tabular}{ll}
\hline APs & RAs \\
\hline AP1 & I Portuária, II Centro, III Rio Comprido, VII São Cristóvão \\
AP2 & IV Botafogo, V Copacabana, VI Lagoa, VIII Tijuca, IX Vila I sabel \\
AP3 & X Ramos, XI Penha, XII M éier, XIII Engenho Novo, XIV I rajá, XV M adureira \\
AP4 & XVI Jacarepaguá, XXIV Barra da Tijuca \\
AP5 & XVII Bangu, XVIII Campo Grande, XIX Santa Cruz \\
AP6 & XX IIlha do Governador, XXI IIha de Paquetá, XXIII Santa Teresa \\
\hline
\end{tabular}

AP.1 - É composta dos bairros mais antigos do município e sua população vem se reduzindo a partir da década de 1980. AP.2 - Compreende a Zona Sul e a Zona Norte, sendo formada pela expansão daquela parte mais antiga do município, contida na AP. 1. Reúne o maior contingente de população de renda elevada.

AP.3 - Estas RAs constituem os subúrbios da Central do Brasil e da Leopoldina, formadas ao longo das vias férreas homônimas. Possuem população densa, estável e os maiores contingentes de população favelada da cidade. AP.4 - As suas duas RAs são extensas áreas territoriais cuja ocupação foi acel erada no final da década de 1960, seguindo-se uma grande expansão. Na RA XXIV - Barra da Tijuca -, há predomínio de segmentos sociais de nível de renda mais elevado, com renda média dos chefes de família de 18 salários mínimos. A RA XVI - Jacarepaguá tem composição heterogênea e renda média pouco superior a cinco salários mínimos. Conta também com grande número de favelas e conjuntos habitacionais destinados à população de baixa renda.

AP.5 - Suas RAs são áreas de expansão urbana recente, com crescimento muito acelerado. Constitui, juntamente com a AP.4, a denominada Zona O este do município. Na AP.5, predominam grandes contingentes de população de renda média entre 2,4 e 3,1 salários mínimos e seu nível de concentração populacional é pouco elevado, ainda existindo áreas com características rurais em seus territórios, embora oficialmente urbanas.

AP.6 - É a de mais difícil apreciação, reunindo áreas descontínuas e muito diferenciadas.

A RA XXI - Il ha de Paquetá, na baía de Guanabara - é voltada para o turismo e tem população muito pequena.

$\mathrm{N}$ ão tendo óbito por tuberculose, foi excluída do estudo.

A RA XX - Ilha do Governador - é uma região de ocupação recente. Seu processo de expansão populacional tem sido contido, devido aos problemas viários de sua condição insular e de dependência de saída rodoviária única, deficiente sistema de transporte hidroviário, cuja operação é ainda bastante recente e a legislação restritiva quanto ao gabarito da altura dos edifícios por causa da proximidade com o Aeroporto Internacional do Galeão. Quanto às condições socioeconômicas, embora na Ilha do Governador sejam encontradas mais de vinte favelas, a renda média foi 6,2 salários mínimos, no ano de 1991.

A RA XXIII - Santa Teresa - é continental e conta com áreas de ocupação antiga do entorno do Centro. Tem apresentado perda e substituição de sua população original por outra de baixa renda, que mora em favelas e cortiços. A renda média é de 5,1 salários mínimos.

\section{Resultados}

Nas figuras 1, 2 e 3, respectivamente, a distribuição por RA dos indicadores CM TB, chefes de família com renda média entre um edois salários mínimos (r 1-2) e número médio de cômodos por domicílio (NMC), são exemplificadas a concordância ea inversão de ten dência dos indicadores sociais com a do CM TB.

Essa forma de apresentação da distribuição dos valores dos indicadores socioeconômicos e o CM TB, referentes ao ano de 1991, oferece uma primeira e objetiva informação visual de sua relação, que no conjunto estudado podem se apresentar como concordância, inversão ou ausência de uma conexão visual evidente. Essa informação foi, depois, confirmada mediante os valores da correlação e sua significância estatística (Tabela 1).

Embora não apresentadas em mapas, a simultaneidade de concordância e a correlação elevada ocorreram também com: índice de Robin Hood (IRH) e razão de renda entre os
$10 \%$ mais ricos e os $40 \%$ mais pobres $(10 \%+$ r). Por outro lado, registram-se a inversão do padrão de distribuição e a correlação elevada com os indicadores: taxa de escolaridade de nível superior (TES); área média por domicílio (AMD); renda média em salários mínimos $(R m)$ e, proporção de chefes de família com renda entre dez e quinze, dezesseis e vinte e mais de vinte salários mínimos ( r10-15, r1620, r20e+).

Os indicadores índice de pobreza (IP), índice de Gini (IG), proporção de renda média dos chefes de família até $1 / 2$ salário mínimo e entre 1/2-1 (r1/2 e r1/2-1) apresentaram correlação alta, mas menos elevada que os anteriores. Os indicadores taxa de anal fabetos (TA), densidade demográfica (DD), densidade de população favelada (DPF) apresentaram baixa correlação estatística com o CM TB. 
Figura 1

Coeficiente de mortalidade por tuberculose por 100 mil habitantes/ano, segundo Região Administrativa, Município do Rio de Janeiro, 1991.

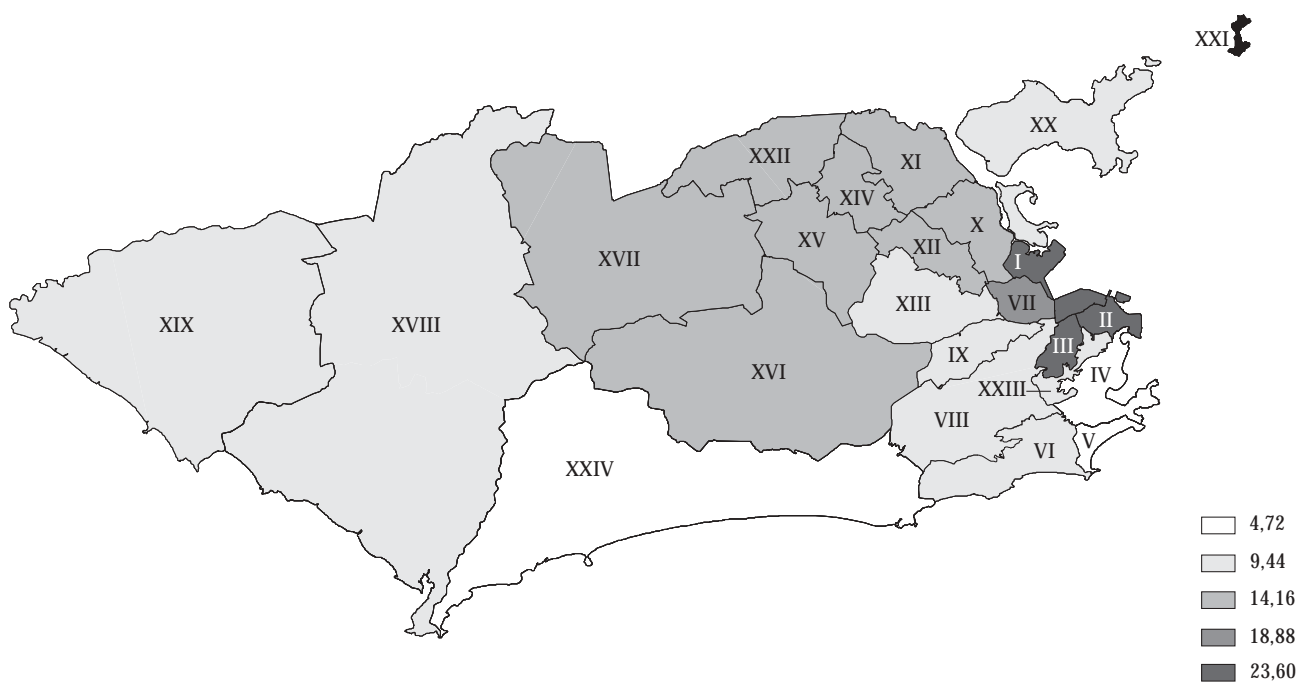

Figura 2

Percentagem de chefes de família que tiveram renda média entre 1-2 salários mínimos, segundo a Região Administrativa, M unicípio do Rio de Janeiro, 1991.

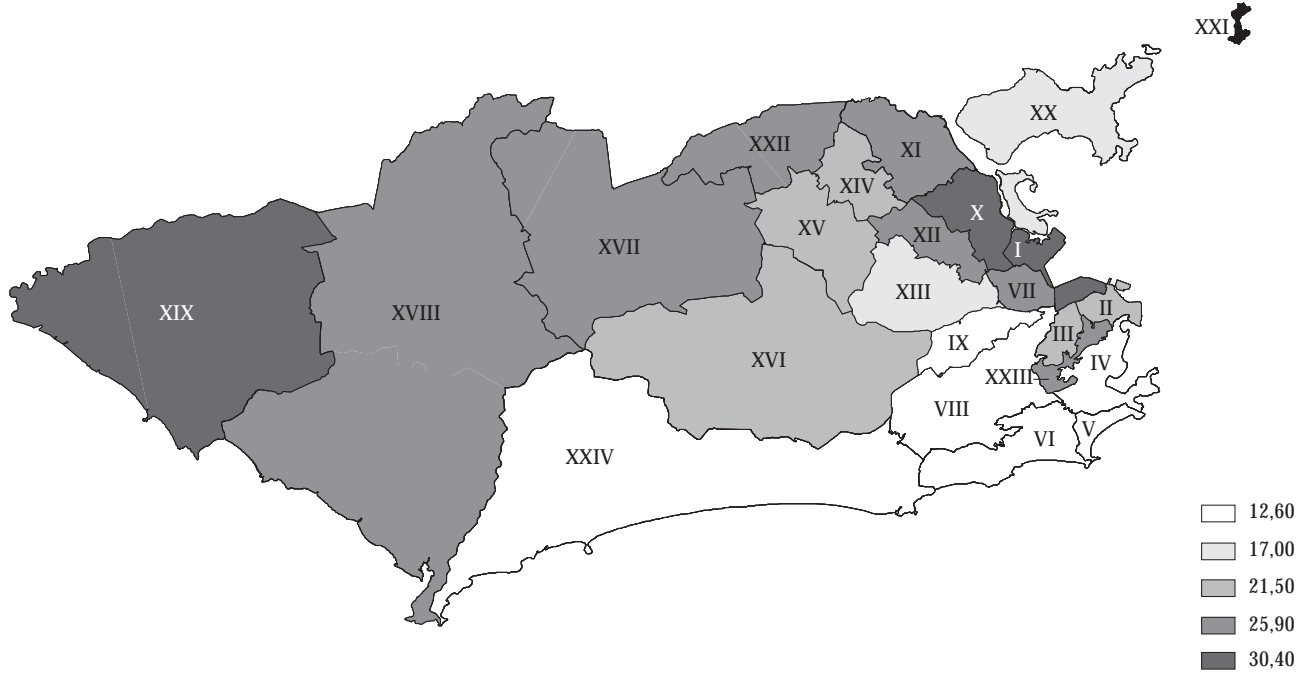

fonte: IPLAN RIO, 1995 
Figura 3

Número médio de cômodos por domicílio, segundo a Região Administrativa, Município do Rio de Janeiro, 1991.

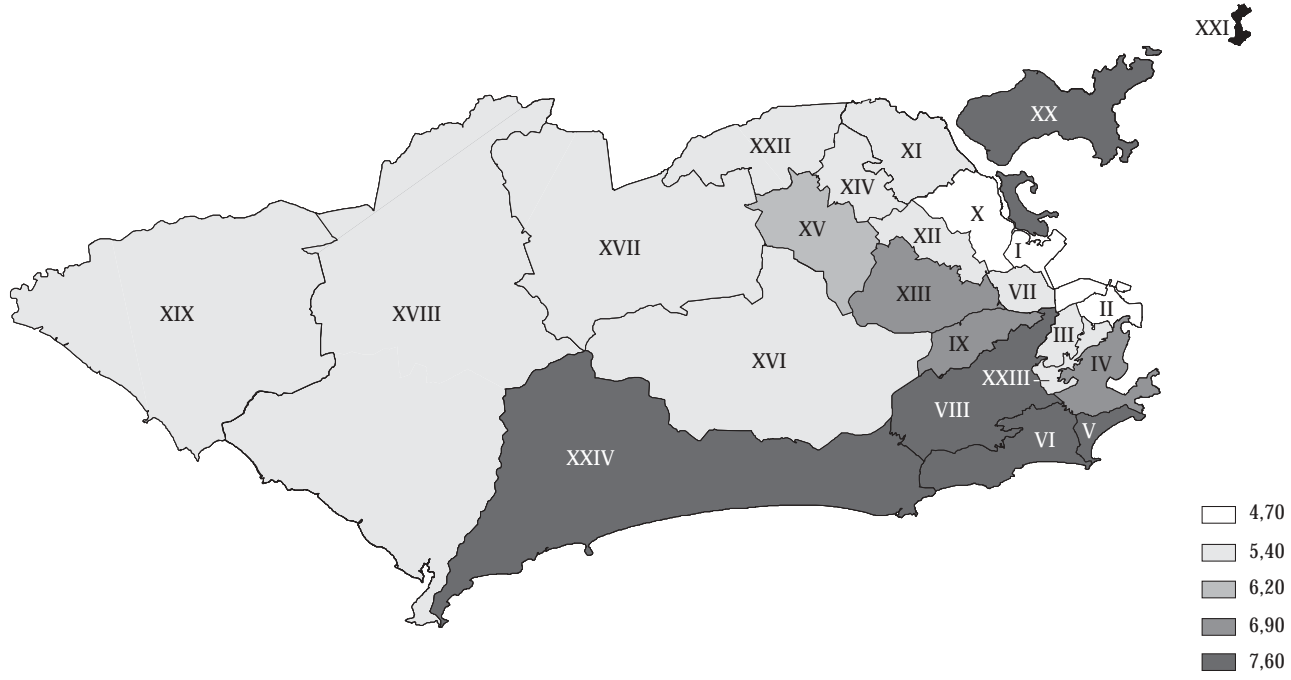

fonte: I PLAN RIO, 1995

\section{Discussão}

0 primeiro aspecto dos resultados a ser apontado refere-se aos indicadores de renda (econômicos). Todos apresentaram forte correlação com o CM TB, embora entre aqueles, os dois indicadores de renda mais baixa ( $r 1 / 2-1$ e r $1 / 2)$ e o índice de Gini foram os que apresentaram os níveis menos elevados de correlação, ainda que altos. Mas, o fato mais relevante foi que no conjunto dos indicadores de renda oito tiveram correlação muito elevada com o CM TB. Desta forma, todos se revelaram sensíveis para detectar o enlace entre a condição socioeconômica e o desfecho desfavorável da tuberculose.

Por seu turno, os indicadores nomeados por sociais se mostraram menos sensíveis, verificando-se que quatro, entre sete, não tiveram correlação muito elevada com o CM TB: a taxa de analfabetos (TA), índice de pobreza (IP), densidade demográfica (DD) e densidade de população favelada (DPF) (Tabela 1). Resultado surpreendente, que demanda considerar cada um, em particular, para explicar a correlação encontrada.

Entre os indicadores sociais, a correlação do IP com o CM TB apresenta valores intermediários. Pode-se explicar esse resultado, como foi apresentado acima para outros indicadores com valores intermediários, por raciocínio probabilístico: trata-se de correlação baseada em dados de um único ano, tendo algumas RAs número pequeno de óbitos prejudica seu desempenho estatístico. Além disso, verifica-se que o IP tem valores expressivos em quase todas as RAs, podendo contribuir para mascarar a expressão estatística da influência das diferenças sociais sobre aspectos determinados do processo saúdedoença.

Outro caso é o da TA, cuja correlação como CM TB foi baixa. Esse comportamento talvez possa ser parcialmente explicado, admitindo-se uma menor influência do analfabetismo sobre o comportamento da evolução clínica eepidemiológica da tuberculose nas condições de uma grande cidade, onde a informação sanitária encontra melhor condição de veiculação. Entretanto, é mais provável que resida principalmente no fato de 0 analfabetismo estar presente em todas as RAs do município do Rio de Janeiro, com taxas não tão diferenciadas. Estas podem ser explicadas pela presença de população favelada e outras de baixa renda, em todas as suas RAs. Reforçam esse raciocínio as correlações expressivas da TA com a DPF e com o NM C sendo, a segunda, inversa. São el evadas, também, as correlações da TA com a maioria dos indicadores de renda, sendo inversa com os de renda alta. 
Tabela 1

Coeficientes de mortalidade por tuberculose,* indicadores sociais, de renda, correlação e valores de p, Rio de Janeiro,1991.

\begin{tabular}{|c|c|c|c|c|c|c|c|c|c|c|c|c|c|c|c|c|c|c|}
\hline \multirow{2}{*}{$\begin{array}{l}\text { R. } \\
\text { Administrativa }\end{array}$} & \multirow[t]{2}{*}{ СМТВ } & \multicolumn{10}{|c|}{ Indicadores Sociais } & \multicolumn{7}{|c|}{ Indicadores de Renda } \\
\hline & & TA & IP & DD & DPF & TES & $M D$ & NMC & $\mathrm{IRH}$ & IG & $\%+r$ & $\mathrm{Rm}$ & $r 1 / 2$ & $r 1 / 2-1$ & $r 1-2 r$ & 5 & 20 & $0 \mathrm{r} 2$ \\
\hline & & & & & & & & & & o & & & & & & & & 0,4 \\
\hline & & & & & & & & & & & & & & & & & & 8 \\
\hline & & 0 & 2,5 & & 38 & 7 & & & 0 & 6 & & & & & & & & 6 \\
\hline & & 2,4 & 5,1 & & & & & & 0,3 & 4 & & & & & & & & 16,9 \\
\hline & & 2,6 & 2,6 & & & & & & & & 10 & & & & 4 & & & \\
\hline & & 7 & 9,7 & & & & & & & & & & & & & & & \\
\hline & & 10,3 & 26 & & & & & & & & & & & & & & & 0,8 \\
\hline & & & & & & & & & & & & & & & & & & \\
\hline & & & & & & & & & & & & & & & & & & 2 \\
\hline & & 13,6 & בc & & & & & & & & & & & & & & & 7 \\
\hline & & & & & & & & & & & & & & & & & & 9 \\
\hline & & 4,1 & & & & & & & & & & & & & & & & 0,7 \\
\hline & & 10 & & & & & & & 0 & & & & & & & & & \\
\hline & & 5 & & & & & & & & & & & & & & & & 4 \\
\hline & & 6,6 & & & & & & & & & & & & & & & & 0,7 \\
\hline & & 7,8 & & & & & & & & & & & & & & & & 3,6 \\
\hline & & 9 & & & & & & & & & & & & & & & & 0,6 \\
\hline & & 10 & & & & & & & & & & & & & & & & 0,8 \\
\hline & & & & & & & & & & & & & & & & & & \\
\hline & & & & & & & & & & & & & & & & & & 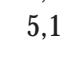 \\
\hline & & & & & & & & & & & & & & & & & & 0,4 \\
\hline & & & $1 L_{1}$ & & 15,6 & 21 & & & & & & & & & & & & 3,3 \\
\hline & & 6,8 & & & 0,8 & 22,6 & 8 & & 0 & 0,4 & 11,7 & 1 & 1 & 5,3 & 10,9 & 2,6 & 9,33 & 30,8 \\
\hline & & & & & $T$ & & & & & & & & & & & & & 3,4 \\
\hline mirelaça & 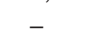 & 0,23 & 0,38 & $-0,13$ & 0,08 & $-0,48$ & $-0,50$ & $-0,72$ & 0,44 & 0,38 & 0,42 & $-0,50$ & 0,41 & 0,38 & 0,50 & $-0,50$ & $-0,54$ & $-0,5$ \\
\hline
\end{tabular}

* por 100 mil habitantes/ano

Fontes: IPLAN RIO, 1995 e Szwarcwald et al., 1999

CM TB - coeficiente de mortalidade por tuberculose; TA - taxa de analfabetos; IP - índice de pobreza;

DD - densidade demográfica; DPF - densidade população favelada; TES - taxa de ensino superior:

AM D - área média por domicílio; N M C - número médio de cômodos por domicílio; IRH - índice de Robin Hood;

IG - índice de Gini; rm - renda média em salários mínimos; $10 \%$ + r - razão de renda média entre os $10 \%$ mais ricos e os $40 \%$ mais pobres;

r1/2-1 - renda média entre 1/2 e 1 salário mínimo; r1-2 - renda média entre 1 e 2 salários mínimos;

r10-15 - renda média entre 10 e 15 salários mínimos; r16-20 - renda média entre 16 e 20 salários mínimos;

r20e + renda média superior a vinte salários mínimos.

A correlação do CM TB com os indicadores DD e DPF também não foi elevada. Sobre o primeiro indicador, cabe dizer que as grandes densidades são encontradas tanto nas RAs com CM TB elevados, como nos de baixa mortalidade por tuberculose. N esta condição está a RA Copacabana que, com a mais alta densidade demográfica, é uma das que tiveram os mais baixos valores do CMTB.

Quanto à DPF, esta apresentou valores elevados em RAs com coeficientes intermediários de mortalidade por tuberculose. Foi o caso da Lagoa (RA VI) a qual, apesar de possuir o segun- do maior nível de renda do município, tem cerca de $1 / 3$ de sua população habitando nas favelas da Rocinha e do Vidigal. Nesta RA, como ocorre também em outras, os coeficientes são formados pela combinação da freqüência de óbitos por tuberculose entre populações nas quais a morte por esta doença é bastante rara e outras nas quais ela é freqüente. Resulta, neste caso, um coeficiente de mortalidade por tuberculose de valor intermediário só suspeitado quando se tem em conta aquela desigualdade social da RA.

A desigualdade social influencia também a formação da maioria dos indicadores socioe- 
conômicos reduzindo sua expressão quantitativa. Por tal razão, quando dados epidemiológicos e demográficos de populações social mente distintas estão associados em uma mesma RA, esses indicadores podem perder sensibilidade para a análise estatística dificultando a explicação epidemiológica com base nos resultados estatísticos. Nesse sentido, corroboram também as características da correlação, cuja expressão é mais influenciada pelos valores mais altos e os mais baixos dos indicadores; isto pode reduzir o número de áreas que pesem na correlação, vindo daí a redução de sua sensibilidade a um resultado não significativo.

Essa forma de composição geográfica de indicadores sociais, quando combina condições em oposição extrema, fica na dependência das proporções e da forma dessa combinação para a manutenção de sua sensibilidade. No caso da densidade demográfica, que mede a concentração de indivíduos em determinado espaço independente da forma como se organizam as moradias, valores elevados podem não significar más condições sociais de vida. E, mais uma vez, o caso de Copacabana (RA V) é exemplar, devido ao fato de a concentração ocorrer no bairro e não internamente ao domicílio, que é o espaço mais fundamental para a transmissão da tuberculose.

Também corroboram com esse raciocínio os indicadores AM D e NMC, que medem a concentração intradomiciliar e expressam meIhor a homogeneidade social das RAs. A mbos tiveram correlação el evada e inversa (Tabela 1) e o segundo apresentou o coeficiente de corre lação mais elevado e negativo. Como o espaço intradomiciliar é aquele que mais reflete a homogeneidade social, tem a maior correlação, indicando a grande sensibilidade e especificidade deste indicador, para apontar áreas de maior risco de ocorrência de óbitos e, possivelmente, de casos de tuberculose.

Sendo, também, indicador de concentração de várias condições sociais de vida desfavoráveis no micro ambiente social e domiciliar, 0 NM C é condensador de várias condições essenciais, que influem sobre a epidemiologia da tuberculose. Expressa, portanto, o grau de carência social que, em última instância, éo componente mais determinante da evolução de um caso de tuberculose para o óbito.

É certo hoje que os ambientes intradomiciliares, mais concentrados e menos arejados, põem em contato estreito e promíscuo as pessoas que os habitam, favorecendo a veiculação de doenças de transmissão aérea, como a tuberculose. Não bastasse isso, neles se concentram populações com muitas outras condições desfavoráveis de vida como desnutrição, menor acesso aos serviços de saúde, etc.

Projetando-se as condições acima, no espaço urbano, são localizadas em áreas antigas de degradação urbana e concentração populacional intensa, como já verificaram, no município de São Paulo, Nogueira (1984) eAlmeida (1990).

No Rio de Janeiro, também as favelas podem contribuir para a sensibilidade desse indicador. Esta afirmativa parece conflitante com o resultado da sua correlação com o CM TB. Entretanto, já foi mencionado que as favelas mais antigas e localizadas nos melhores bairros do Rio de Janeiro têm melhor organização, contam com alguns serviços urbanos, embora deficientes, e possuem parte de suas habitações já transformadas em moradias com espaço interno ampliado. Pode-se dizer que esses indicadores (NM C e AM D), por medirem efetivamente a concentração a que a maioria da população de determinada área está submetida em seu ambiente cotidiano principal, contêm uma condição intrínseca de reunir vários componentes de vida social que podem ter forte influência sobre a transmissão e a mortalidade por tuberculose (seu valor é inverso), podendo aumentar sua sensibilidade. Levando-se o raciocínio para níveis microorganizacionais da estrutura social, talvez se possa afirmar que na casa onde houve uma morte por tuberculose é maior o risco de ocorrerem outros eventos fatais pela doença.

Essa característica de um indicador, que Ihe agrega sensibilidade, parece ter sido verificada também no estudo de D'orsi e Carvalho (1998), quando relaciona o índice de parto cesáreo com o nível de escolaridade materna e encontra uma correlação positiva e elevada. Este resultado contraria a lógica técnica e científica de indicação daquele procedimento. Entretanto, como é 0 caso do NM C para a tuberculose, reúne efeitos de outros componentes na determinação do processo saúde-doença, no caso, a indicação de cesárea ligada aos interesses socioeconômicos.

0 indicador TES apresentou correlação inversa e elevada. Portanto, quanto mais elevado o indicador, melhores as condições de vida, sendo esperados menos casos e menos óbitos por tuberculose. Estes últimos não somente pela menor proporção de casos, mas também por menor letalidade.

Este indicador parece reunir, como se evidencia no município do Rio de Janeiro, carac- 
terísticas que lhe conferem vantagem intrínseca. Os indivíduos com maior escolaridade são os que auferem renda mais alta, habitam meIhores e mais saudáveis residências. Na geografia urbana, concentram-se em determinadas áreas, onde, mesmo quando existem favelas ou outras formas de habitação de populações de baixa renda, estas não são as que apresentam as piores condições. Como resultado, o indicador em pauta pode apresentar baixa correlação inversa com a mortalidade por tuberculose.

0 desempenho estatístico dos indicadores sociais é mutuamente influenciado mediante a atuação de múltiplos componentes da vida social, que atuam nas condições reais e se entrelaçam na determinação social da doença. Por esta razão, tais indicadores podem operar como variáveis de confusão, e no planejamento práticas podem dificultar a própria atuação de controle da doença, principalmente quando são pouco valorizados na programação.

Além dos indicadores analisados, especificamente sociais, há outros que se constituem problemas sanitários fortemente associados, como al coolismo, desnutrição, doença mental e outras doenças, em especial a Aids, que podem desempenhar importante papel na determinação dos índices de mortalidade. Estes também, quando detectados, podem transformarse em indicadores e em problemas objetivos, como fatores de risco a serem controlados. Para muitos destes, a atribuição da principal responsabilidade na redução de seus efeitos no controle da tuberculose não pode deixar de ser conferida ao âmbito institucional do programa de controle da doença. Tal atribuição opera, portanto, como indicadora de sua abrangência, na mesma medida que o desempenho como indicador de sua eficiência.

Os demais indicadores estudados foram agrupados como "indicadores de nível de renda" e estão na tabela 1, onde são incluídos os resultados da correlação. São os indicadores econômicos, mas todos se embutem de forte conotação social, por se proporem a medir concentração de renda em populações determinadas.

Entre estes, o índice de Gini (IG), a percentagem de chefes de família com renda média até meio salário mínimo ( r1/2) e a percentagem de chefes de família com renda média entre meio e um salário mínimo (r1/2-1) apresentaram correlação intermediária. Este comportamento pode ser explicado apenas por raciocínio probabilístico. Tal possibilidade é considerada por que o CM TB se comporta estatis- ticamente como um evento raro. Assim, a correlação feita com dados de mortalidade de apenas um ano, pode coincidir em várias RAs com valores de menor expressão, os quais a influenciam reduzindo-a.

Entretanto, a explicação para esse comportamento pode ser buscada na distribuição social muito heterogênea que ocorre no Rio de Janeiro, no espaço urbano, e em duas outras questões que devem ser consideradas. Em primeiro lugar, existe uma grande massa de população em condições intermediárias, mas não muito favoráveis quanto às condições de vida. Ou seja, há uma proporção muito pequena de ricos para uma muito elevada de pobres, que se distribuem em quase todas as RAs do município do Rio de Janeiro. Este grande contingente populacional atua na composição dos indicadores, reduzindo a expressão de seus valores mais extremos, tornando-os menos sensíveis.

Possivelmente, os resultados da correlação com todos os indicadores de cunho predominantemente econômico sofrem as perturbações já mencionadas da heterogeneidade social, geográfica, e da grande desproporção entre ricos e pobres, resultando em proporções de valores menos elevados. M esmo assim, todos os demais indicadores de renda tiveram correlação elevada, e, embora não tanto quanto à do número de cômodos por domicílio, corroboram na demonstração do enlace entre os planos socioeconômico e biológico no desenvolvimento do processo de saúde-doença estudado.

Chama atenção o fato de os indicadores que apresentam valores mais bem definidos e coerentes com a expressão social predominante na $\mathrm{RA}$, serem aqueles que produzem os maiores níveis de confiança da correlação com o CM TB. $\checkmark$ indo reafirmar o estreito laço de determinação das condições socioeconômicas sobre a tuberculose (morbidade e mortalidade).

\section{Conclusões}

O estudo mostrou um evidente predomínio de freqüências e coeficientes mais elevados de mortalidade por tuberculose nas RAs onde predominam populações de baixa renda.

Entre os indicadores sociais, o número de cômodos por domicílio foi o indicador mais sensível para revelar (de modo inverso) o enlace entre as esferas social e biológica, no desenvolvimento da tuberculose e na sua mortalidade, seguindo-se a área média por domicílio tam- 
bém em correlação inversa. Tal fato mostra, também, que esses indicadores podem ter particular importância para revelar a homogeneidade/heterogeneidade social, em áreas urbanas definidas. M ostra, ainda, que a grande concentração humana no espaço intradomiciliar é uma expressão direta da pobreza e baixa condição de vida. Portanto, representa papel relevante nas características de gravidade que pode assumir o processo particular de saúde-doença da tuberculose. A observação destes indicadores sociais no espaço urbano pode contribuir na revelação das maiores concentrações da pobreza e permitir, ainda, a identificação de outros componentes desfavoráveis da sua vida social contribuintes nos riscos de desenvolver formas graves da doença, e/ou morrer em conseqüência da mesma.

Conclui-se, também, que a quantificação da correlação entre indicadores sociais e o CM TB (biológico) pode indicar qual deles concentra maior poder de determinação sobre a tuberculose. E, por conseguinte, quais componentes da vida social produzem efeitos mais favoráveis, ou mais desfavoráveis, para o desenvolvimento do processo de morbidade determinado e seu desfecho mais grave, o óbito.

0 entrelaçamento da ação desses indicadores faz com que operem em conjunto e interajam mutuamente entre si, como variáveis in- tervenientes, cujos efeitos não é possível isolar. Estes somente podendo ser adequadamente apreciados mediante a análise epidemiológica.

Os indicadores de renda, embora revelassem níveis menos elevados de correlação (enlace entre os dois níveis do processo: social e biológico), são mais diretos em expressar a condição socioeconômica de uma população dada e se apresentam, em sua maioria, em correlação significativa com o CM TB. Este comportamento dos indicadores econômicos mostrou a determinação da condição econômica sobre a vida social, podendo-se inferir que determinam também o padrão e o perfil epidemiológicos, constituídos ao nível de sociedades ou grupos sociais economicamente distintos.

Como se pode inferir pelo comportamento de indicadores sociais, principalmente o NMC eAMD, é no terreno social que se materializam as condições para o desenvolvimento das formas particulares de doenças, como a tuberculose e suas formas graves que conduzem mais freqüentemente ao óbito.

Pode-se concluir, também, que as limitações da ação do sistema público de saúde em localizar precocemente os indivíduos de mais baixa condição social que desenvolveram a tuberculose, e tratá-los eficazmente, vêm sendo um fator relevante na elevada mortalidade que ocorreno município do Rio de Janeiro.

\section{Referências bibliográficas}

Albuquerque AFR \& Rodrigues BA 1952. Evolução secular da mortalidade por tuberculose no Distrito Fede ral. Revista Brasileira de Tuberculose (144):725-789.

Almeida M M M B 1990. Vigilância epidemiológica da tuberculose no município de São Paulo: uso de dados de mortalidade. Tese de doutorado. Faculdade de Saúde Pública da USP. São Paulo, 95pp.

Antunes JLF, Waldman EA 1999. A tuberculose através do século: séries temporais para a mortalidade em São Paulo, Brasil, 1900-1997. Cadernos de Saúde Pública 15(3):463-476.

Bates B 1994. Bargaining for life: a social history of tuberculosis, 1876-1938. University of Pennsylvania Press, Filadélfia, 435pp.

Bertolli Filho C 1997. História social do tuberculoso, perspectivas documentais. IV Encontro de História e Saúde. Rio de Janeiro.

Black D, M orris SN, Smith C, Townsend P 1982. The pattern of present health inequalities, pp. 51-64. In Townsend \& Davidson (orgs.). Inequalities in health: the black report. Penguin Books, Nova York.
Bradford Hill A 1965. Princípios de estadística médica. “El Ateneo" Editorial, Buenos Aires, 365pp.

César CLG 1990. Fatores de risco associados à mortalidade infantil em duas áreas da região metropolitana de São Paulo, 1984-1985: proposta de instrumentos pre ditivos. Revista de Saúde Pública 24(4):300-310.

D' Orsi E \& Carvalho MS 1998. Perfil de nascimentos no município do Rio de Janeiro: uma análise espacial. Cadernos de Saúde Pública 14(2):367-380.

Dubos R \& Dubos E 1952. The white plague. Little Brown and Company, Boston, 277pp.

Engels F\& Marx K 1985. Prefácio à edição inglesa de “A condição da classe operária em Inglaterra", vol. III: pp. 490-505. In F Engels \& K M arx. O bras escolhidas. Progresso, Lisboa.

[FIBGE] Fundação IBGE 1982. Censo demográfico: Resultados preliminares - Rio de Janeiro. V.1, tomo 4, n.18. (9o Recenseamento Geral do Brasil), Rio de Janeiro.

[FIBGE] Fundação IBGE 1993. Censo demográfico: resultados preliminares - Rio de Janeiro. V. 1, n. 20. (10o Recenseamento Geral do Brasil), Rio de Janeiro. 
Guimarães R 1985. Determinação social e doença endêmica, o caso da tuberculose pp. 211-233. In PEC/ENSP/ ABRASCO. Textos de apoio: epidemiologia I. PEC/ ENSP/ABRASCO, Rio de Janeiro.

IPLAN RIO 1980. Informações básicas da Cidade do Rio de Janeiro: 1979. Rio de Janeiro.

IPLAN RIO 1981. Informações básicas da Cidade do Rio de Janeiro: 1980. Rio de Janeiro.

IPLANRIO 1982. Informações básicas da Cidade do Rio de Janeiro: 1981/82. Rio de Janeiro.

IPLANRIO 1983. Regionalização dos serviços de saúde de interesse municipal na Cidade do Rio de Janeiro. Rio de Janeiro.

IPLAN RIO 1984. Informações básicas da Cidade do Rio de Janeiro: 1981/84. Rio de Janeiro.

IPLANRIO 1991. Anuário Estatístico da Cidade do Rio de Janeiro: 1990. Rio de Janeiro.

IPLANRIO 1992. Anuário Estatístico da Cidade do Rio de Janeiro: 1991. Rio de Janeiro.

IPLANRIO 1993. Anuário Estatístico da Cidade do Rio de Janeiro: 1992/93. Rio de Janeiro.

IPLANRIO 1995. Anuário Estatístico da Cidade do Rio de Janeiro: 1993/94. Rio de Janeiro.

Lima TA 1996. Humores e odores: Ordem corporal e ordem social no Rio de Janeiro, século XIX. Revista História e Ciências da Saúde II(3):44-94.

Mckeown T \& Lowe CR 1981. Evaluación de mejoras en salud, pp. 17-35. In T Mckeown \& CR Lowe. Introducción a la medicina social. Siglo veintiuno Editores, M éxico (DF).

M inistério da Saúde/CENEPI/SIM 1998. Sistema de informação de mortalidade 1979 a 1997, dados de declaração deóbito. [CD ROM ]. Braśllia (DF): M S/DATASUS.

Nascimento DR 1991. Tuberculose: de questão pública à questão de Estado: a liga brasileira contra a tuberculose. Dissertação de mestrado. Instituto de M edicina Social da UERJ, Rio de Janeiro 144pp.

Nogueira PA 1984. Tuberculose como causa de óbito em adultos residentes no M unicípio de São Paulo em 1980. Tese de doutorado. Faculdade de Saúde Pública da USP, São Paulo, 91pp.

Penna M LF 1988a. Uma abordagem ecológica da tuberculose. Cadernos de Saúde Pública 4(4):356-362.
Penna M LF 1988b. Tuberculose: assistência ou controle: revisão dos dados que apóiam a existência de efetividade epidemiológica dos programas de controle baseados no diagnóstico e tratamento de casos. Boletim da CNCT 2(1):5-14.

Penna M LF 1988c. Tuberculose: assistência ou controle: discussão sobre a efetividade epidemiológica de programas de controle baseados no diagnóstico de tratamento de casos. Boletim da CNCT 2(2):5-14.

Pereira JC \& Ruffino N etto A 1985. Saúde-doença e sociedade: a tuberculose - o tuberculoso, pp. 237-248. In PEC/ ENSP/ABRASCO. Textos de apoio, epidemiologia I. Rio de Janeiro.

Ribeiro L 1956. A luta contra a tuberculose no Brasil. Rio de Janeiro: apontamentos para sua história. Editorial Sul Americana S.A, Rio de Janeiro, 402pp.

Ruffino Netto A 1975. Epidemiologia da tuberculose: estudo de al guns aspectos ligados a model os de prevenção, diagnóstico e modelos epidemiológicos, pp. 1-34. Tese de livre-docência. Faculdade de M edicina Ribeirão Preto da USP, Ribeirão Preto.

Ruffino Netto A \& Pereira JC 1981. M ortalidade por tuberculose e condições de vida. Saúde em D ebate 12:27-34.

Snow J 1967. Sobrea maneira de transmissão da cólera. USAID, Rio de Janeiro, 161pp.

Spiegel M R 1985. Estatística (2a ed.). M cGraw-Hill do Brasil, São Paulo, 454pp.

Stead WW, Senner JW, Reddick WT \& Lofgren, JP 1990. Racial differences in susceptibility to infection by Mycobacterium tuberculosis. New England Journal of Medicine 322(7): 422-427.

Szwarcwald CL et al. 1999. Desigualdade de renda e situação de saúde: 0 caso do Rio de Janeiro. Cadernos de SaúdePública 15(1):15-28.

Vicentin G 1991. Saúde e mineração na Amazônia: o caso da Mineração Rio do Norte. Dissertação de mestrado. Escola Nacional de Saúde Pública, Fundação O swaldo Cruz, Rio de Janeiro, 274pp.

Vicentin, G 2000. Evolução da mortalidade por tuberculose no município do Rio de Janeiro, 1979-1995. Tese de doutorado. Faculdade de Saúde Pública da Universidade de São Paulo, São Paulo, 159pp.

Vieira S 1985. Introdução à bioestatística (2a ed.) Campus, Rio de Janeiro, 294pp.

Artigo apresentado em 10/11/2001

Versão final apresentada em 4/3/2002

Aprovado em 20/3/2002 